\title{
GALL-SICKNESS OF SOUTH AFRICA. (ANAPLASMOSIS OF CATTLE.)
}

\author{
By Dr ARnold Theiler, C.M.G., Government Veterinary
} Bacteriologist to the Transvaal Government.

IN a previous number of this Journal ${ }^{1}$ I published an article on $\mathrm{A}$ New Trypanosoma and the Disease Caused by It. I stated there that in the course of the hyperimmunisation of cattle against rinderpest a disease was repeatedly noted of which the causal factor was thought to be the trypanosome. It will be remembered that it was noted as a result of blood injections into animals immune and susceptible to rinderpest. I further stated that the disease described by Hutcheon as jaundice or biliary fever ${ }^{2}$ was probably identical with the one I had observed. This latter was first of all noticed by Spreull in cattle which had been inoculated with defibrinated blood. Hutcheon stated that he himself saw it on Robben Island in cattle which had been inoculated with virulent blood and serum. It was commonly called gall-sickness. Owing to the fact that in South Africa many ailments in cattle are designated by that name, it becomes evident that a great difficulty has always been experienced in determining the malady which was originally meant under that name. Here, at least, we had something tangible; we injected blood containing the parasite into cattle, and typical lesions appeared in several instances, namely, those of jaundice, which latter is responsible for the name gall-sickness, and hence the identification of the parasite with the disease.

It was already noted by me at that time that the trypanosome could be present in cattle without causing any illness at all. The trypanosome was later also found outside South Africa, generally accidentally, associated and unassociated with other distinct diseases. This led to the opinion that it was usually a harmless parasite.

In South Africa at that time we only knew of one other blood parasite which could be transmitted by the inoculation of blood, namely, piroplasma bigeminum. The pathological lesions caused by that organism, namely, hæmoglobinuria, were considered so typical that they could not be mistaken for the disease which was thought to be caused by trypanosoma theileri.

In the course of later investigations I found a spirochæte which when injected into cattle caused a fever, and the blood sometimes showed distinct lesions of oligocythæmia subsequent to this injection. I then differentiated a new small piroplasm (piroplasma mutans) which I could transmit by inoculation from immune to susceptible cattle. This parasite appeared after a rather long incubation period, which was followed by fever and severe lesions of oligocythæmia, polychromasia, basophilia, and the formation of normo- and megaloblasts. These lesions were identified with the presence of piroplasma mutans alone, because, as a rule, they appeared at the same time.

The conclusion from these facts is that in South Africa the injection of blood taken from an ox selected at random may trans-

1 “Jour. of Comp. Path. and Therap.," Vol. XVI., Part 3.

2 Hutcheon: "Annual Report of the Chief Veterinary Surgeon, Cape of Good Hope," 1897. 
mit three different parasites-piroplasma bigeminum, piroplasma mutans, and spirochate theileri. With the inoculation of blood containing trypanosoma theileri any of these may have been transmitted and complicated the infection. In the article referred to I distinguished an acute disease with a pathological change of the red corpuscles, and the disease without any such change. These changes, in the form of basophilia, were minutely described, and it was then definitely decided that they were by no means typical for the trypanosome, but also occurred with piroplasma bigeminum, and cases were enumerated in which the interference of trypanosoma theileri could be excluded.

We now know that after a blood injection piroplasma bigeminum appears with a very short period of incubation, lasting four to five days; and as soon as the acute fever is over, which lasts for about three to six days, basophilia puts in an appearance and remains for some time. We also know that the inoculation of immune blood transmits piroplasma bigeminum; and, notwithstanding the fact that this parasite may be absent on microscopical examination, basophilia may appear quite typically subsequent to the injection.

The incubation period of trypanosoma theileri is also a short one, slightly under or perhaps just over that of piroplasma bigeminum. Therefore, when we inject blood of a South African born ox-that is to say, an animal immune against redwater - into a susceptible beast, we also inject piroplasma bigeminum, and although the presence of this parasite may not subsequently be diagnosed in the blood smear yet the resulting basophilia is due to its presence. It is in this way that we have to explain at least some of the acute diseases observed after blood injections. It must be stated here that this explanation only holds good for the experimentally produced cases. In practice we occasionally found infection with trypanosoma theileri accompanied by all the lesions of basophilia, but usually complicated with piroplasma bigeminum and also with spirochæte theileri. These latter two are present in the immune animal, and the re-appearance of the bigeminum must also be put down to the accidental infection with trypanosoma theileri, causing a breakdown in immunity. The reappearance of piroplasma bigeminum would then be the cause of the anæmia which is diagnosed as gall-sickness.

Basophile granulations were noticed not only after a short incubation period of piroplasma bigeminum but also after long intervals. Some of the acute cases related by me only occurred after a long period of incubation. On account of the absence of any other blood parasite, such as piroplasma bigeminum, they were also considered to be trypanosoma infections, due to the fact that the anamic condition, together with the pronounced basophilia, corresponded with that found after the short incubation period. Anæmia and basophilia after long incubation periods are not observed in inoculated redwater, nor can they be explained by the presence of trypanosoma theileri, and we have to look elsewhere for the reason.

As already stated, in those days we did not know of the existence of piroplasma mutans. This is a parasite which requires a long period of incubation, varying from twenty to forty days, before it can be detected in the blood. We have described blood lesions typical of oligocythæmia, with pronounced basophilia. as a result of the 
infection with this parasite; and for these reasons, although no animal under observation died, we identified this disease with gallsickness, also thinking that the anæmia was due to this parasite.

Ever since I separated piroplasma mutans as a specific parasite I have drawn attention to the appearance of peculiar coccus-like bodies found on the periphery of red corpuscles, which I have described under the name of "marginal points." I registered their presence whenever they were met with, and they will be found mentioned in all my investigations on piroplasma mutans. In my report for I905-06 I made special reference to the presence of marginal points occurring in the experiments which I had described just previously. A tabulated resumé was given showing the number of days which elapsed between the injection of blood and the appearance of these marginal points; the shortest period was noticed to be twenty-three days, and the longest time thirty-seren days, the average being about thirty days. The injected animals were classified in three groups:-

First.-Piroplasma bigeminum and marginal points appearing in one and the same animal.

Second.-Piroplasma bigeminum appearing alone.

Third.-No piroplasma bigeminum, but marginal points.

The animals of the second group were selected to be injected. with blood of an ox which in all previous experiments had constantly produced marginal points. In this instance after the blood injection no marginal points were noted, and it was pointed out that the inference to be drawn from this experiment was that a previous injection of redwater blood would give immunity against marginal points, and that therefore the marginal points seemed to be connected with the development of piroplasma bigeminum. Owing to the insufficient number of animals experimented on, I did not draw this conclusion definitely, but left the question open. The fallacy in the experiment was that the animals concerned might have had immunity against both marginal points and piroplasma bigeminum, assuming that the marginal points were independent parasites. In my second article on piroplasma mutans ${ }^{2} I$ made repeated reference to the appearance of marginal points in the injected animals without paying any further attention to their presence. In the third contribution on the same subject ${ }^{3}$ the conclusion arrived at was that "in some animals the presence of marginal points has been noticed. I am not yet in the position to explain the nature of these points or to state the relation in which they stand to piroplasma mutans." To understand this conclusion, it must be remembered that the strain of piroplasma mutans was considered to be a pure one, unassociated with any other parasite, particularly piroplasma bigeminum: and, accordingly, the marginal points represented an element of uncertainty, since previously one was inclined to identify them with piroplasma bigeminum. The question as to their true nature had therefore to be left open.

The marginal points also interfered to a great extent in an experiment undertaken for the purpose of testing cattle on their immunity

\footnotetext{
1 "Annual Report, Govt. Vet. Bac., Transvaal," 1905-06, p. 6.5.

2 "Jour. of Comp. Path. and Therap.," Tol. XX., pp. 1-18.

Ibid., Vol. XXIr., p. 133.
} 
against redwater. These were six Ayrshire heifers injected in London by $\mathrm{Mr}$ Stewart Stockman with blood of an immune redwater animal. Two of the heifers had been injected with the English strain of redwater, two with the South African strain, and two with both strains. The South African strain of redwater had been sent by me to London previously in ticks. Soon after their arrival in the Transvaal the six heifers were exposed to natural infection. The result was that all animals showed severe fever, during which the lesions of a grave anæmia were pronounced. And in these three animals - two of which died subsequently-marginal points were registered. The conclusion at that time was that the inoculated animals could not withstand the infection with ticks, which were very prevalent at that particular season of the year (summer), and that accordingly the immunity against redwater had broken down.

The foregoing observations show ( $I$ ) that marginal points appeared in cattle injected with blood taken at random from a South African born animal which had recovered from redwater; (2) that marginal points were noted to complicate an apparently pure infection of piroplasma mutans; and (3) that these marginal points appeared in cattle which were undoubtedly immune against redwater by reason of a previous inoculation.

An interpretation of these facts is only possible when we consider the marginal points as a parasite sui generis, in no way related to either piroplasma bigeminum or piroplasma mutans, but, like these parasites, present in immune animals. With blood from such animals it was transmitted by injection together with piroplasma bigeminum and piroplasma mutans, and against these two piroplasms it did not give any immunity. I had held this idea for a considerable time, but I had not been able to give such proofs as were necessary to remove all doubts. This became possible in the new series of experiments again undertaken for the purpose of testing the immunity of cattle inoculated in England against South African redwater.

On the I 8 th November I 908 ten Sussex heifers were inoculated in London by Mr Stewart Stockman. They all showed redwater reactions, some with symptoms of hamoglobinuria, and piroplasma bigeminum was present in all but one case, but this latter animal nevertheless proved its immunity when tested subsequently whilst still in England. Accordingly, when the heifers were despatched to South Africa there was no doubt that they were immune against redwater. They had been under Mr Stockman's observation for forty-one days, from the I 8 th November to 29th December; they were then shipped, and arrived in Pretoria on the 22nd February I909. I decided not to expose them to the natural tick infection, as was done with the previous lot of animals, but to test their immunity by a second injection of redwater blood, in the hope that in this way the immunity would be improved.

For this purpose the ten heifers were divided into two equal lots, and each lot was injected with the blood of a different animal. The result was that in no instance was there any breakdown of immunity against piroplasma bigeminum. Some of the animals showed a slight reaction, during which piroplasma bigeminum was present, 
but in such rare numbers that it was by mere chance that their presence was detected. The conclusion could be safely drawn that the inoculation in England protected the animals from redwater when they were injected for the second time in the Transvaal. Subsequent events proved to be very interesting. With the exception of one heifer, between the twenty-seventh and thirty-second days after the blood injection a distinct fever reaction ensued, and this was either preceded, or shortly afterwards succeeded, by the appearance of the typical chromatic bodies hitherto described as marginal points. The number of these points increased with the progress of the fever, and the temperature fell when their number decreased or when they disappeared. Some time after the chromatic points had made an appearance the lesions of oligocythamia, anisocytosis, poikilocytosis, polychromasia, and basophilia, as well as normo- and megalo-blasts, were noted. The animal which in the first instance did not become infected did so when subsequently injected with the blood of a sick animal. Of the ten animals in the experiment five died. It is important to note that during the whole illness no red urine was passed, and none was found on post-mortem examination. This new evidence to my mind was sufficiently conclusive to warrant one considering the marginal points as the cause of this disease, as a parasite independent of the piroplasma bigeminum, and as an organism dissimilar from any hitherto known in protozoology.

The next inference to be drawn is that the ticks which I sent to England represented a pure infection of piroplasma bigeminum. If marginal points stood in any relation to piroplasma bigeminum their appearance would have been noted in England, not only in the last lot of cattle, but also in the many others treated in a similar way by Stockman, ${ }^{1}$ 'Fadyean, and Nuttall. ${ }^{2}$ If, therefore, the marginal points represent an independent parasite, it must be possible to separate it from any other parasite, especially piroplasma bigeminum. Considering, however, the short incubation period of this latter parasite and the long one of marginal points, it becomes evident that the latter cannot be separated by inoculation from the former if they are both present in the same animal. The reverse is, however, possible ; and I succeeded in isolating piroplasma bigeminum by inoculating the blood collected at the commencement of the redwater reaction into a susceptible English heifer, when piroplasma bigeminum only appeared. Subsequently this animal was tested with blood containing marginal points, and again, after a typical incubation period, these parasites appeared. Finally, by inoculating a susceptible English heifer with blood of a Cape Colony animal obtained from Aliwal North-the animals from which district always prove to be susceptible to redwater - an infection was obtained which was quite pure and not associated with any other blood parasites. The same results were again obtained in a later experiment.

Taking all these facts into consideration, no other conclusion can be arrived at except that these marginal points are the cause of a typical disease - a grave anæmia which to a great extent resembles that caused by piroplasma bigeminum, that they stand in no relation to piroplasma bigeminum, and that they represent a new genus of 
protozoon, for which I have proposed the name "anaplasma," the species under consideration to be called "anaplasma marginale." 1

Accordingly, the disease which would be called "anaplasmosis" is the one noted in all my previous experiments, and recognised under the name gall-sickness by South African farmers. I transmitted it at the same time with my trypanosome, spirochæte, and piroplasma mutans inoculations when it was contained in the blood of the animals which showed the parasite under investigation, and in this way my previous experiments find an explanation. Attention must, however, be given here to the fact that not all diseases in which grave anæmia as mentioned above are found is due to anaplasmosis. Redivater is the first exception. Gall-sickness and redwater resemble each other to such an extent that the farmers constantly call them the "sister diseases." The gall-sickness due to the presence of trypanosoma theileri observed under natural conditions now finds an explanation; under the influence of this parasite a revival of either anaplasma marginale or piroplasma bigeminum may occur and acute anæmia result. The work on piroplasma mutans will have to be done over again; it was complicated with anaplasma marginale, and the anæmic lesions found in the injected animals were due to the presence of the latter parasite.

On the other hand, not every disease called gall-sickness by the South African farmer is anaplasmosis. As mentioned before, this name principally indicates a disturbance of the digestive organs with lesions of the liver and alterations of the bile. These are, however, the most pronounced lesions on post-mortem examination in piroplasmosis and anaplasmosis, and accordingly we may safely state that anaplasmosis represents the genuine gall-sickness, since the piroplasmosis is usually accompanied by hæmoglobinuria, and this symptom permits of the differentiation.

THE LITERATURE ON ANAPLASMA MARGINALE.

\section{Texas Fever.}

The parasite which I have called anaplasma marginale is well known in literature. Smith and Kilborne, ${ }^{2}$ in their work on Texas fever, distinguish two forms of that disease, first the acute disease, and secondly the mild type of Texas fever. They further distinguish two forms of parasites: first, the pyriform and fusiform bodies, the post-mortem forms of which are roundish; and, secondly, the peripheral coccus-like bodies. The American investigators divide the cycle of piroplasma bigeminum into three different stages: (a) the (hypothetical) swarming or mobile stage (intraglobular); (b) the stage of the peripheral coccus-like body; and (c) the stage of the larger forms (pyriform and spindle-shaped bodies). These authors state as follows: ". . . After the (hypothetical) swarm spore has penetrated into the corpuscle it comes to rest, loses its bright refrangent appearance, and it attaches itself near the periphery of the corpuscle as a pale body, which is only detected

I Theiler : “Transvaal Medical Journal," Vol. V., No. 6, January 1910.

3 Smith and Kilborne: "U.S. Department of Agriculture Eighth and Ninth Annual Reports of the Bureau of Animal Industry," 1891 and 1892. 
with difficulty in the unstained corpuscle. This body next undergoes division, which is probably incomplete, for in the more advanced stages the two resulting bodies are as a rule attached to each other. These remain close together while the infected corpuscle is circulating in the blood. This stage of the coccus-like body, like the preceding hypothetical stage, must be regarded as recognisable because of a retarded development of the micro-parasite. It is probable that this retardation of development in susceptible animals is due to meteorological conditions, such as low temperature of the air, and to partial immunity. In acute attacks the enormous multiplication of the parasite in the blood shows how rapid in such cases its development is, and how ephemeral these intermediate stages must be. The period of retardation may vary in length, but it seems probable that this stage may remain in circulation at least several days. . . . The two coccus-like bodies resulting from division begin to grow, and assume fusiform outlines, etc."

There cannot be any mistake in the interpretation of this statement; it is clear that the peripheral coccus-like bodies are considered to be the stage preceding the pear-shaped one. When in acute cases, in which this latter is only met with, the former ones are not seen, then it is because the development from coccus into pearshaped body takes place very rapidly.

Smith and Kilborne, however, already had some doubt as to the true nature of these bodies. They say: ". . . If we admit their parasitic nature as highly probable we have still the question before us whether they are stages of the Texas fever parasite or of another parasite transmitted with it." They then proceed to prove that these bodies belong to the cycle of piroplasma bigeminum. The following statement is of particular interest to us. "Two cows inoculated with blood from healthy North Carolina cattle early in July I 892 developed the acute type of Texas fever, with the appearance of pyriform parasites within the red corpuscles. Both recovered, and the number of corpuscles was rising towards the normal when, at the end of August, a relapse was detected in both animals. The number of corpuscles was rapidly falling again, and many were infected with the coccus-like bodies. Re-infection from without can hardly be considered in these cases, as there were no ticks in the field, and tivo control animals had normal blood throughout the season." The interpretation from my point of view is, that in July the animals were inoculated with blood of an immune animal which contained two parasites-piroplasma bigeminum and anaplasma marginale. The former, with its shorter incubation period, appeared first, the latter came later. The periods stated correspond exactly with the incubation period and length of the disease as found in South Africa.

In this instance it is clear that the peripheral coccus-like body appeared after the pyriform one. In perusing the appendix to the report, where individual cases are given, we notice, for instance, in No. 49 the appearance of large parasites in the first instance, and at a later period the peripheral coccus-like bodies.

Case No. 56 represents an animal exposed in the first year without showing any disease; in the following year it was again exposed, and contracted a disease in which pyriform bodies were found at the 
commencement of the illness, but at the height of the fever the coccus-like bodies infested 30 per cent. of the corpuscles, and at the end only one pair of large parasites were found. The former instance refers to the typical course of the infection by the two parasites inoculated simultaneously, as explained before; and the latter one refers to an animal immune to piroplasma bigeminum - the first infection having passed unnoticed-and not immune to anaplasma marginale. The fact that only one pair of piroplasma bigeminum was noticed supports this view. If the peripheral coccus-like bodies could develop into pyriform ones, we would expect that of the 30 per cent. infected corpuscles a much greater number would appear.

In all these instances Smith and Kilborne had to do with mixed infections, but, according to their statement, they also have seen pure infections of anaplasma marginale. They say, “... an outbreak produced after the middle of September I 889 developed cases containing the coccus-like bodies only. In one of these cases lilled in a dying condition the spleen and the liver were affected as in acute cases, but hamoglobinuria was absent."

As already explained, Smith and Kilborne considered the appearance of peripheral coccus-like bodies as being mainly due to the influence of cold, and the disease in which they were found was called the autumnal form. These cases were grouped as follows :-

First.-Animals exposed to Texas fever late in the season (October and November).

Second.-Animals which had passed through an acute attack earlier in the summer. (Second attack or relapse in October and November.)

Third.-Animals which contract a mild disease during or previous to the season of the acute disease.

Smith and Kilborne add regarding No. I, "in the first group the disease is mild and miy pass unnoticed . . . rarely a corpuscle with a pair of large pyriform bodies is detected." Accordingly, we have to deal here with animals immune to piroplasma bigeminum, probably owing to a previous mild attack of a pure infection which was followed by an outbreak of anaplasmosis when the animals were exposed later in the year. The American authors started their experiments in July, August, and later, and therefore it is evident that in view of the long period of incubation the disease would appear later in the season. This explanation also applies to' the second group. The third group consisted of animals which showed either the anaplasma infection first and the piroplasma infection later, or in which both infections appeared at the same time, which may have been due either to a pure infection of anaplasma marginale in the first instance, succeeded by a piroplasmosis infection which happened to be so timed that both parasites appeared simultaneously, or, what is more likely, the appearance of anaplasma marginale may have caused a breakdown of the immunity against piroplasma bigeminum, so that parasites increased in numbers and became very prevalent.

Accordingly, there is nothing inconsistent in Smith and Kilborne's observations with my conception of both pyriform and coccus-like bodies. I may state here that an experiment of mine has shown 
that anaplasma marginale is transmitted by the tick boophilus decoloratus, as had to be concluded from all the evidence quoted hitherto; and that, similar to what is noted in the case of piroplasma bigeminum, the incubation period lasts longer after tick infection than after blood injection. This fact may aid in the understanding of the cases noted by Smith and Kilborne later in the season. From my South African experience, the season has nothing to do with the appearance of coccus-like bodies; on the contrary, the summer is the time when we met with the greatest number of anaplasma infections.

The conclusion at which we arrive is, that the term Texas fever is a collective name for two different diseases, caused by two distinct parasites; the one is due to piroplasma bigeminum, and corresponds to our redwater, the other to anaplasma marginale, and identical with our gall-sickness.

\section{Tristera.}

This disease was studied in the La Plata States in 1904 by Knuth. ${ }^{1}$ The cause of this disease is, as was already known before, piroplasma bigeminum. Knuth also saw the peripheral bodies, which he met frequently, and usually after the piroplasma bigeminum infection. His description of these points corresponds to that of the North Americans and to mine. He did not see them in animals from countries free from ticks, but only in animals after natural or artificial infection. One observation which he quotes is of particular interest. In one animal he first saw the piroplasma bigeminum infection, and forty-one days later, when the animal appeared to be ill, showing a high fever, he again examined the blood and noticed the peripheral points, which were so frequent that from two to four points were seen in one and the same corpuscle. The animal died after an almost complete dissolution of blood corpuscles, accompanied with the appearance of many punctate cells (basophile granulations). A second animal which had been inoculated with the same sample of blood showed these parasites forty-six days later, after which time they gradually disappeared.

When the peripheral bodies were present in the blood of the former animal it was tapped and the blood injected into six fresh ones, four of which could be considered to be immune against tristeza, whereas two were not. These two animals developed peripheral bodies, but not piroplasma bigeminum. On the tenth and eighteenth days after injection the punctiform parasites were very frequent, and on the twenty-eighth day basophile granulations became very noticeable.

Knuth considers these points to be stages in the cycle of piroplasma bigeminum. The facts given by Knuth correspond with those noted by me. The inoculation of immune blood first gives rise to piroplasma bigeminum, and then to anaplasma marginale. The fact that after the injection of sick blood no piroplasma bigeminum appeared, although the animal had recovered from an infection of this parasite, may be accidental, as it is not every cubic centimetre of immune blood which contains parasites, and this has repeatedly been found in the transmission experiments of redwater by different investigators.

3 P. Knuth : "Experimentelle Studien ueher das Texas fieber der Rinder." 
We therefore come to the conclusion that, as with the term Texas fever, we have to understand under the name tristeza two different infections-piroplasmosis and anaplasmosis.

\section{The Cachectical Piroplasmosis of the Transcaucasus.}

Luhs and Dschunskowsky in their article Die piroplasmosen der Rinder ${ }^{1}$ give a description of tropical piroplasmosis, of which they distinguish an acute and a cachectical form, the former being due to a small piroplasm, now called piroplasma annulatum. In describing the cachectical form they give a description of the pathological lesions, which correspond with those found in gall-sickness and they lay particular stress on the degeneration of the liver and other parenchymatous organs and the presence of clear urine.

The cause of the cachectical form, according to the two Russian authors, are the peripheral bodies, of which they give a description corresponding with ours. It is noteworthy that notwithstanding the fact that these two scientists have seen piroplasma bigeminum in the Transcaucasus they do not identify the peripheral body with the cause of redwater, but with piroplasma annulatum, of which they think it represents a more resistant form. Apparently Dschunskowsky and Luhs must either have had to do with cattle immune to piroplasma bigeminum infection and not immune to anaplasma, or they were dealing with a pure infection of the latter, one which complicated their piroplasma annulatum infection.

According to my conception, the cachectical form of tropical piroplasmosis of Dschunskowsky and Luhs has no connection with the latter disease, but represents anaplasmosis.

\section{The Hemoglobinuric of Europe.}

This disease is due to piroplasma bovis, a parasite at least closely related to piroplasma bigeminum if not identical with it. I consider that if the peripheral bodies belong to the cycle of piroplasma bigeminum, as Smith, Kilborne, and Knuth state, we must find them in the cycle of piroplasma bovis. As far as I am aware this has never been described in England or on the Continent. In perusing the work Ueber die Hamoglobinuvie der Rinder in Deutschland, by Kossel, Schüt, Weber, and Miessner,' we notice the records of prolonged observations, long enough to notice the appearance of such striking parasites as anaplasma marginale with the resulting anamic lesions, if such had been present. There is only one interpretation of these facts, and that is that Europe knows only of a pure infection of piroplasma bovis, probably identical with piroplasma bigeminum, and accordingly the disease corresponds with our redwater.

Wherever the associations of piroplasma bigeminum and anaplasma marginale have been noted in North and South America, in the Transcaucasus, and in Africa, the transmitting agent can be identified with a certain genus of tick, namely boophilus. In North and South America it is boophilus annulatus, in the Transcaucasus boophilus calcaratus, and in South Africa boophilus decoloratus. This genus.

1 "Centralbl. f. Jakter. Parasit. and Infekt.," Bd. XXXV., No. 4, Jan. 1904.

2 "Arbeiten aus. dem. Kaiserlichen Gesundheitsamte.," Vol. XX., 1904. 
is absent in Europe, and that may perhaps account for the absence of anaplasmosis.

\section{DESCRIPTION OF THE IISEASE.}

Anaplasmosis is a disease of cattle, due to a protozoon-anaplasma marginale-which invades and destroys the red blood corpuscles, causing primarily an acute anæmia (oligocythæmia) accompanied by a high fever, and secondly a degeneration of the larger parenchymatous organs. Recovery from the disease gives resistance to subsequent infections. The immune animals remain a reservoir for the virus. The blue tick (boophilus decoloratus) acts as host or transmitter of the disease.

Cause. - The cause of anaplasmosis is a protozoon of a new genus -anaplasma, species marginale-which differs from any protozoan organism at present known inasmuch as it consists entirely of chromatic substance-a nucleus, plasmatic substance, as far as can be ascertained, being absent.

The parasite has hitherto been described as peripheral " coccus-like bodies" or "marginal points," and has always been considered to be of a protozoan nature, mainly due to the fact that it takes the typical chromatin tint when stained by any of the Romanowsky modifications. Its shape is that of a round or oval body, certainly resembling a coccus. Occasionally round the body a polar zone is noted, the globule apparently not staining as well here as elsewhere.

The multiplication of this parasite seems to take place by fission. In many cases, when the number of parasites increases rapidly, double forms are met with, either separated and placed closely together, or as splitting forms in which the two bodies have not yet completely separated. As a rule, these parasites are situated on the periphery of corpuscles, sometimes reaching over the margin; they are also seen more towards the centre of the corpuscles, but these cases are not so frequent.

Anaplasma marginale multiplies rapidly in a susceptible animal, and invades a large percentage of corpuscles, amounting to about 50 per cent. in severe cases, and then three or four are frequently met with in one corpuscle.

The blood lesions which are thus caused, and hence also the severity of the disease, stand in a certain relation to the number of parasites.

Geograplical Distribution.-Anaplasmosis has been seen in various parts of the world. It was first seen and described in North America, although it was not there recognised as an independent disease. It is present in South America, as shown by Knuth. It is found all over Africa. I have mentioned its cxistence in the Transvaal ever since I undertook the examination of blood smears on a large scale, and its frequency is shown in our annual returns of examinations. I saw it in blood smears sent to me from Rhodesia. Recently Spreull ${ }^{1}$ found it in the Eastern Province of Cape Colony. Pitchford informed me that he saw it frequently in Natal. I have seen it both in British East Africa and in Uganda. Balfour, in one of his 
Reports, ${ }^{1}$ mentions its existence in the Soudan. It is present in the Transcaucasus, as Dschunskowsky and Luhs have shown. I do not know whether it has yet been found in India, where redwater, which it usually accompanies, exists, although in various parts of Asia piroplasma mutans has been seen as an accompaniment of redwater.

Sitsceptibility of Species of Animals, Breed, Age, etc.-So far, anaplasmosis has only been noticed in cattle. Experiments designed to transmit the disease by inoculation of blood of an animal suffering from an acute attack have failed to produce any reaction in a freshly imported Argentine horse, all of which are highly susceptible to equine piroplasmosis, and in a merino sheep of South African origin.

The susceptibility of cattle varies greatly. We can distinguish an absolute non-susceptibility on the one side and a high susceptibility on the other. The former is represented by the old native cattle, born and bred in infected areas, and the latter by the freshly imported high-bred pedigree stock. So far, the observations point to the fact that all cattle born in South Africa are susceptible to anaplasmosis, but the calves recover easily from the disease, and it passes almost unnoticed whilst they are very young.

This statement is based on a more recent experiment, in which calves of Africander cows were after birth immediately placed in a tick-free stable and reared on milk. They were injected with blood containing anaplasma marginale; they contracted the disease in due course, and, although the microscopical examination revealed a very grave disease, which was clinically evidenced by the paleness of all mucous membranes, the animals easily recovered. It must be stated here that the inoculation of blood also caused the appearance of piroplasma bigeminum, which appeared first and made no impression on the animals. This observation may account for some of the diseases known as "kalverziekte," of which it is stated that the liver on post-mortem is found to be very yellow, because we have to accept that in practice where the sick animals are at the same time exposed to obnoxious influences in the environment not all animals recover. Further, it may also account for the stunted growth of some of the calves which have been more or less severely infected during an earlier period of their life. In this respect the effects of the two diseases, piroplasmosis (redwater) and anaplasmosis, on the red corpuscles must interfere with the normal growth of the young animal. Although, as a rule, a calf born in South Africa passes through anaplasmosis early in its life, yet there are exceptions, judging both by the observations in the field and by those experimentally obtained. The former are usually made after moving cattle from higher altitudes to lower, or from one part of the country to another, and we can imagine without any difficulty that there are areas in which the infection is present to a slight extent only; accordingly, not necessarily all cattle become infected whilst calves. But this is especially the case with cattle which are born and bred in stables and in paddocks which are kept more or less tick-free.

Anaplasmosis is observed in South African cattle of almost any age, and the explanation just given does not explain all these cases.

1 Wellcome Research Laboratories, Khartoum, Second Report. 
What is known about the virulency of redwater applies similarly to this disease. There exists a difference in the virulency of certain strains of anaplasma ; and although, generally speaking, the recovery from a first attack of the disease confers a high degree of resistance, it does not give complete immunity. Thus in former inoculation experiments $I$ have seen that the inoculation of blood from an animal gave rise to the appearance of the disease, and when the animal which supplied the blood was injected in turn with blood from a different animal it also developed the infection. This fact gives another explanation of the observations already alluded to, and will account for cases in which the affected animals show only a mild infection, the parasites being met with in small numbers.

The imported English pedigree cattle show the greatest susceptibility, as experiments will show, the morbidity averaging about Ioo per cent., and the mortality amounting to over 50 per cent. of the infected cattle. It is not yet certain to what extent this high mortality is influenced by youth, but the animals in my observations averaged about eighteen months old.

\section{THE VARIOUS FORMS OF ANAPLASMOSIS AND THE LESIONS.}

The disease, whether produced by injection of blood or by tickinfection, has a typical incubation period, the length of which seems to be influenced by various factors. It seems to be shorter in cases caused by blood inoculation than by tick infection (so far only one exact observation regarding ticks is to hand). In the cases caused by blood injection it seems to make a difference whether an animal has been injected with immune blood (blood which does not show any parasites under the microscope) or by virulent blood, which shows the parasites present. It varied between twenty-seven and thirty-two days in the former, and lasted for about sixteen days in the latter.

(a) Anaplasmosis Ending with Death.-After an incubation period of twenty-seven and twenty-three days respectively the fever reaction started. In some instances the anaplasma was noted before the onset of the fever. In all cases the parasites increased rapidly in numbers and the number of red corpuscles decreased, the remaining corpuscles becoming quite pale and colourless. In the shortest cases death occurred about the seventh day after the onset of the fever. The lesions of polychromasia and basophilia were not present in the cases of shortest duration.

In cases of death occurring at a later period (eight to twelve days) after the appearance of anaplasma, polychromasia and basophilia were constantly noticed for some days (four, six, and seven days before death). In these instances normoblasts also made their appearance.

(b) Anaplasmosis Ending with Recovery.-In cases that recovered the usual period of the fever reaction lasted from fourteen days to three weeks. The temperature reaction was very high. The parasites increased in numbers, but after a while seemed to remain stationary; they usually disappeared either before or at the same time as the temperature returned to normal, but sometimes they remained even longer. About eight days after the appearance of anaplasma 
the blood lesions of polychromasia and basophilia put in appearance; they were succeeded a few days later by normoblasts. The blood did not return to normal conditions for a considerable length of time.

(c) Acute Anaplasmosis Ending in Recovery.-There were some instances in which the course of the disease ran very quickly, ending with recovery, both the typical and high fever reaction only lasting about seven days. The parasites did not seem to increase to such grcat numbers, and they decreased again with the drop of the temperature. This was eventually succeeded by the appearance of polychromasia and basophilia; rare normoblasts were seen in one instance.

(d) Mixed Infections.-Mixed infections were noticed with piroplasma bigeminum and with piroplasma mutans. Piroplasma bigeminum appeared either before the anaplasma infection or during the reaction. Piroplasma mutans usually appeared during or after the anaplasma reaction. The picture of anæmia, as described before, was present in all instances and well pronounced.

Clinical Symptoms. - The febrile reaction is the first clinical symptom noticed, and high fever is present for some days before the animal is actually observed to be ill. Capricious appetite or refusing to feed is therefore the first symptom which will attract attention. The severity of these symptoms varies, of course, with the susceptibility of the animals. Calves, as a rule, do not show such high fever, and continue to feed, although sometimes but sparingly. In full-grown cattle these symptoms are generally more pronounced, more particularly in imported stock. A careful observer will soon notice that the sick beasts lose in condition, and the wasting is sometimes so rapid that a marked difference becomes visible within one day.

At this period the symptoms of anæmia become very pronounced.

The muzzle appears pale, the sclera of the eye is quite white, the conjunctiva is bloodless, and the mucous membrane of the mouth loses its pink colour. All severe cases in calves and adults begin like that, and in animals which die late or recover the parts mentioned take a slight yellow colour, which may become very pronounced. A yellowness of the skin can then be noticed where it is transparent, such as in the ears or where the hair is not thick, along the flanks, in the region of the udder, and between the hind legs. An oczasional abrasion of the skin has quite a yellow surface.

The intestinal tract also becomes involved. There is first a slight constipation. Later the fæces lose their normal colour and take a brownish-yellow stain. Diarrhœa, with evacuations of yellow fæces intermixed with mucus, is occasionally observed. The urine is distinctly yellow, but never blood-stained. As the disease advances the animal frequently lies down, and finally it remains in the recumbent position.

Both the heart's action and the respiration become hurried, and a peculiar groaning noise accompanies every breath. This state of affairs, which indicates the approaching end, is followed by muscular tremblings in the flanks and shoulders.

Nervous symptoms are sometimes noticed shortly before death, such as spasms of the extremities and the head, and sometimes the animal becomes very aggressive. 
In cattle which recover the symptoms appear in the same order, and may be as pronounced as in an animal which is going to die. Generally, however, the sick animal soon resumes feeding again. The wasting continues for some time. The paleness and the icteric condition only wear off gradually. Convalescence is a slow process.

Diagnosis. - The clinical picture thus described is by no means typical for anaplasmosis. It applies also to cases of redwater. When the acute attack is over the hremoglobinuria is either absent or has passed unnoticed, and the symptoms of anamia and jaundice, however, become pronounced. Here also the approach of death is characterised by similar symptoms.

Microscopically, the picture of anæmia is identical. The lesions of anisocytosis, poikilocytosis, polychromasia, and basophilia, and the presence of normo- and megalo-blasts, are also found in the sequelæ of redwater. Whereas, however, in redwater as a rule piroplasma bigeminum has altogether disappeared when the above symptoms are noted, the anaplasma marginale is present for a longer period and at a time when all the anamic lesions are well pronounced. But also in anaplasmosis a stage exists (after the temperature has returned to normal) when all blood parasites are absent.

The presence of anaplasma marginale may give rise to a recrudescence of piroplasma bigeminum, and then we find both parasites together. The reverse may also take place, and, although the piroplasma bigeminum infection is generally the primary one, it will, as a rule, be difficult or even impossible to say so with certainty. Piroplasma mutans may also be found, especially in inoculated animals which have been injected with blood taken at random from any animal born and bred in the country.

Pathological Anatomy.-Animals which died of an acute attack were found in good condition, although a noticeable wasting was observed during the few days of illness. On post-mortem examination the presence of fat deposits was apparent, these being generally of a distinctive yellow colour. The subcutaneous tissue was also of a yellowish discoloration, and occasionally in some parts a gelatinous infiltration was noticed, as, for instance, on the neck, sternum, and abdomen.

The flesh had a pale bloodless colour, and looked as if it originated from a carcase bled to death.

The blood was thin and watery, poor in corpuscles, and did not stain.

In some instances the pleural cavities contained an abnormal amount of liquid, in one instance about a litre.

The pericardium was either found empty, or else contained only clear straw-coloured liquid, and in only one instance was the maximum quantity of $30 \mathrm{cc}$. found to be present.

The lungs were found in a condition of half or full inspiration; there was emphysema of the anterior lobes in one instance. The colour of the lungs was pale yellow, and the existence of an odema was indicated either by the presence of some foam in the bronchi and trachea or by an interstitial infiltration. In one instance the parenchyma was dry.

The bronchial and mediastinal glands were usually slightly 
swollen, soft to the touch, and bloodless. In one instance there was also found a hæmorrhagic infiltration of the lymph sinus.

On the epicardium were found petechiæ, ecchymoses, and hæmorrhagic suffusions. The ventricles were found with and without coagulated blood. The left endocardium was whitish and showed some ecchymoses. The right endocardium was found normal in most cases, in others it showed some ecchymoses. The myocardium was pale and of soft consistence.

The liver was in every instance enlarged; its minimum weight was found to be 6.5 kilogrammes, and the maximum 8.6 kilogrammes. It had a swollen appearance, rounded margins, and a yellow colour, and the sections showed either a brown-yellow or a saffronyellow tinge. The cut surface was granular and had a peculiar glossy shine. The organ also contained but little blood.

The gall-bladder contained inspissated, viscid, dark green bile.

The periportal glands were pale.

The spleen was enlarged in every instance; its minimum weight was found to be $1 \cdot 4$ kilogrammes, and the maximum 3.3 kilogrammes -average $2 \cdot 2$ kilogrammes; its length varied from 55 to $68 \mathrm{~cm}$., and the width averaged $20 \mathrm{~cm}$. The capsule was distended and sometimes showed ecchymoses. The pulp was soft, protruding on section, and the colour was deep red to black; in one instance it was of the consistence of jam, in another firm elastic. The trabecula had disappeared, and the malpighian bodies were swollen.

The third stomach usually contained dry food. In only two instances was food found in the fourth stomach; usually liquid was present, and the mucosa was slightly swollen. There were flakes of mucus present in two instances, and a patchy hyperamia in another, but otherwise the mucosa was found to be pale.

The mucosa of the duodenum was usually bile-stained. The mucosa of the jejunum and ileum was pale, bile-stained, and slightly swollen, and it also showed some red streaks.

The cæcum was either empty or contained bile-stained contents, and the mucosa was found to be pale and folded; the apex showed a patchy hyperæmia.

The colon had yellow-stained food; the mucosa was slightly swollen, and pale or yellow.

The mesenteric glands were swollen, pale, and soft.

The kidneys were easily taken out of their capsule; the parenchyma was bloodless and pale yellow.

The urinary bladder was found to be empty in one instance; it usually contained clear straw-coloured urine.

The brain was pale and so bloodless that the grey substance appeared almost white.

The bone-marrow of the diaphyses was soft, of a gelatinous consistence, and yellowish in colour.

Transmission of the Disease.-This takes place by means of the blue tick. I have so far only one experiment to that effect, but it is convincing and admits of no other interpretation. In this instance the incubation period was very long, and it remains to be seen whether this is the rule or the exception. In this respect it resembles redwater, where the incubation period of the tick-transmitted disease is longer than in the inoculated one. 
It is probable that anaplasma marginale and piroplasma bigeminum can be transmitted not only by the same species of ticks but also by one and the same individual. This, however, remains to be proved, for although one lot of ticks taken off infected cattle transmitted both diseases it is possible that amongst these some were infected with anaplasma only, and others with piroplasma only. It will further be interesting to determine whether other species of South African ticks will transmit the anaplasma, as is occasionally the case with piroplasma bigeminum. Since the blue tick (boophilus decoloratus) is a one-host tick, it follows that the anaplasma infection passes through the egg, as is known to occur with piroplasma bigeminum.

The Virus Reserioir- - All the experiments-both inoculation and tick-transmission-have proved that the immune animals act as the reservoirs of the virus. From these the ticks obtain the virus and re-infect the cattle, and in this respect both anaplasma marginale and piroplasma bigeminum behave in exactly the same way.

This fact explains the constant infection of a veld both for anaplasmosis and piroplasmosis.

Immunity.-Animals which have recovered show a great resistance to re-infection. There is, however, no complete immunity present. An animal may be successfully inoculated more than once, although the second inoculation will only cause slight reaction. This second reaction may be due to a difference in the strain of the anaplasma. The primary reaction gives sufficient ground-immunity to protect an animal against severe lesions or death from a subsequent infection. Further, the disease may reappear under the influence of any other disease, although, as a rule, it does not then take a very acute course, and the blood examinations do not show large numbers of parasites. In this respect it resembles piroplasma bigeminum, piroplasma mutans, and spirillum theileri infections.

Complications arith oller Diseases and Mixed Infections... It is evident from my observations that immunity against redwater in no way influences the susceptibility against anaplasmosis. Under the condition of an inoculation or tick infection the agent of redwater -the piroplasma bigeminum-appears first, and the animal goes through the distinct redwater attack. After it has recovered the incubation period of the anaplasmosis has elapsed, and then gallsickness makes its appearance, and may cause the animal's death. Generally speaking, this second disease was considered to be only a relapse of redwater. Indeed, since the immune animal retains the piroplasma bigeminum in its blood a recrudescence of this parasite may occur, and then the microscope shows the double infection of anaplasma marginale and piroplasma bigeminum. In some cases this recrudescence of piroplasma bigeminum may lead to the reappearance of red urine-a sure sign of the redwater infection. And what happens with piroplasma bigeminum may also occur with piroplasma mutans, so that an animal at a given period of its illness may show these parasites in its blood as well. This is the reason why it was difficult in the past to identify the clinical and pathological lesions found in a sick animal with any of the parasites present.

Preventiz'e Treatment.-The great majority of animals born and 
bred on South African veld become immune against anaplasmosis (and also piroplasmosis) as they grow up. This is due to the fact that the disease does not attack them so severely as it does the older and fully grown animals. The experimental inoculation of susceptible calves has a similar result, and it seems that the younger the animal the better the disease is borne. This, at least, is the case with South African calves born of the Africander or cross-bred stock. It remains yet to be seen whether the same is the case with the imported thoroughbred pedigree stock of Europe. Probably it will also be so, and the practical deduction would be to import pedigree stock as young as possible, at least under one year old, if one wishes to save the greatest possible number both from redwater and sall-sickness.

Against redwater we can inoculate cattle almost safely in England, and the animals retain their immunity when arriving here, but piroplasmosis (redwater) does not protect against anaplasmosis (gallsickness), and for this reason the inoculated cattle will contract the second disease when exposed to the veld. Pedigree stock for South Africa, and we can state for Africa generally. must have immunity against the two diseases before their importation can be accompanied by the best results.

There is reason to hope that this object will also be achieved.

\title{
HEREDITARY UNSOUNDNESS IN HORSES.
}

\author{
NOTES ON EVIDENCE AS TO TIIE HEREDITARI CHARACTER OF \\ CERTAIN PATHOLOGICAL CONDITIONS CONSTITUTING UN- \\ SOUNDNESS IN HORSES (PRINCIPALLY OSSIFICATION OF THE \\ IATERAL CARTILAGES-SIDEBONE), FURNISHED BY EXAMINA- \\ TION OF 2636 CASES. $^{1}$
}

By S. S. Cameron, M.R.C.V.S., D.V.Sc. (Melb.), Chief Veterinary Officer, Department of Agriculture, Victoria, Australia.

\section{PRESENT LACK OF RECORDED EVIDENCE.}

OSSIFICATION of the laterdl cartilages of the foot of the horse, commonly known as "sidebone," has been somewhat indefinitely classified as an "hereditary unsoundness" by various writers during the last half century. On the other hand, a number of authors, and perhaps the majority of breeders, have strenuously denied the influence of heredity as a causal factor, alleging that the ossification develops as a result of external injury or the unequal incidence of concussion brought about by the use of calkin shoes. Arguments, and experiences of isolated instances, have been relied on by both sides, and no definite proof has been given whereby the doubts of the contenders might be set at rest. It does not seem to have been

\footnotetext{
1 One of four contributions to a thesis presented to the University of Melboume for the degree of Doctor of Veterinary Science, November 1909.
} 